\title{
Experiencias educativas en el contexto universitario latinoamericano, María Lourdes Piñero Martín, Francis Carolina González Pérez, Elba Francisca Ávila Perozo Barquisimeto: UPEL IPB, 2021. 362 p.
}

\author{
Edwin Roger Esteban Rivera \\ Dr. en Ciencias de la Educación \\ Universidad Nacional Hermilio Valdizán \\ Huánuco, Perú \\ edroer@gmail.com \\ Sonia Fiorella Callupe Becerra \\ Mg. en Investigación y Docencia Superior \\ Universidad Nacional Hermilio Valdizán \\ Huánuco, Perú \\ cardozo.carlos@uni9.edu.br
}

Cite como:

(ABNT NBR 6023:2018)

RIVERA, Edwin Roger Esteban; BECERRA, Sonia Fiorella Callupe. Experiencias educativas en el contexto universitario latinoamericano. María Lourdes Piñero Martín, Francis Carolina González Pérez, Elba Francisca Ávila Perozo. Barquisimeto: UPEL IPB, 2021. 362 p. Dialogia, São Paulo, n. 38, e20073, maio/ago. 2021. Resenha, p. 1-5. Disponível em: https://doi.org/10.5585/37.2021.20073.

American Psychological Association (APA)

Rivera, E. R. E., \& Becerra, S. F. C. (2021, maio/ago.). Experiencias educativas en el contexto universitario latinoamericano.

María Lourdes Piñero Martín, Francis Carolina González Pérez, Elba Francisca Ávila Perozo. Barquisimeto: UPEL IPB, 2021. 362 p. Dialogia, São Paulo, 38, p. 1-5, e20226. https://doi.org/10.5585/37.2021.20073.

El mundo entero reconfiguró su forma de vida, de socializarse, de realizar las actividades cotidianas, también las modalidades de desarrollar el proceso enseñanza y aprendizaje en todos los niveles educativos por efecto de la COVID-19.

Las universidades latinoamericanas y del mundo entero se vieron forzadas a migrar a una educación virtual, migración que no fue homogénea. Algunas no tuvieron inconvenientes en adaptarse porque contaban con infraestructura tecnológica adecuada y recursos humanos preparados; en tanto, otras aún tienen dificultades para garantizar la formación adecuada de sus 
estudiantes vía on line. Es decir, cada universidad tuvo experiencias particulares para desarrollar las actividades curriculares en contextos de COVID-19.

Experiencias educativas de varias universitarias de América, durante el confinamiento social por COVID-19, son expuestas en Experiencias educativas en el contexto universitario latinoamericano. El libro editado por María Lourdes Piñero Martín, Francis Carolina González Pérez y Elba Francisca Ávila Perozo, connotadas docentes de la Universidad Pedagógica Experimental Libertador Instituto Pedagógico De Barquisimeto “Luis Beltrán Prieto Figueroa”, Venezuela, consta de doce capítulos, cada capítulo ofrece un artículo, sobre los cuales se comenta.

Ricardo Antonio Cristi López y Adolfo Antonio Quinteros Cerpa en Decadencia de la teoría del capital bumano en los sistemas de educación superior latinoamericanos, cuestionan la teoría del capital humano que, se constituyó en un corpus teórico de mayor influencia en el diseño y estructuración de los sistemas educativos modernos, caracterizado por una mirada economicista de la educación. Bajo esta mirada, según los investigadores, "la educación es concebida como un bien económico o de mercado y no como un derecho social” (p. 30). En la educación superior de América Latina se evidencia en la segmentación de la oferta educativa, accediendo a instituciones de calidad, preferentemente los sectores de mayores recursos económicos.

Rosaura Guerra Oñate, Doris Magaly Colina Sánchez y Melissa Paola Sosa Ochoa, nos comparten Factores prosociales y reflexión autocrítica en las actitudes violentas de jóvenes universitarios, de Rosaura Guerra Oñate. Narran la experiencia de reflexión autocrítica en las actitudes violentas de jóvenes estudiantes de la Universidad Popular del Cesar, Colombia. Desde la perspectiva cualitativa, con características de una investigación-acción, generaron cambios en los estudiantes que se tradujo en la disminución de los comportamientos agresivos. A decir de las autoras, "se logró la orientación en un proceso que permita que el universitario se comprometa consigo mismo y con su yo interno para que estos cambios se reflejen en su entorno" (p. 34).

La Responsabilidad Social Universitaria (RSU) también tiene lugar en el libro, Tógliatty Toro de Jiménez, en RSU como gestión universitaria: estrategia de gestión, pedagogía y didáctica. Interculturizar lo bumano, considera que "los problemas ontológicos latinoamericanos son un nudo crítico para la constitución de un sujeto social profesional responsable” (p. 84) y que, una verdadera política de responsabilidad social universitaria debe tener una efectiva práctica cultural que rompa con la colonialidad del ser.

Ana Jacqueline Urrego, Gabriela Rivas Urrego y Mayra Riera Montenegro, desde la perspectiva metodológica cualitativa nos presentan La praxis pedagógica en el contexto de tres universidades del Ecuador: una aproximación desde diferentes áreas profesionales. A pesar que no precisan el nombre de 
las universidades, se sabe que son universidades que cuentan con las facultades de Ciencias Humanas y Educación, Ciencias de la Ingeniería Aplicadas, Ciencias Administrativas. El estudio reafirma que, así como hay docentes por vocación, otros asumieron la docencia porque se les presentó una oportunidad de trabajo; sin embargo, en ambos casos manifiestan estar comprometidos con la mejora de la praxis docente. Los docentes entrevistados consideran a la praxis docente desde tres miradas: como acto pedagógico y didáctico, acto comunicativo y de interacción, y como acto experiencial y cambiante. Al igual que en otros estudios, se evidencia que al docente le costó más adecuarse al trabajo virtual con relación a los estudiantes, pero ahora piensan que es una buena opción para optimizar el proceso enseñanza y aprendizaje.

Por su parte, Milagros Morales Rangel nos ilustra en COVID-19 impacta educación remota y psicología estudiantil, como las universidades en Estados Unidos, específicamente en el Estado de Florida, desplegaron esfuerzos en la implementación de plataformas digitales para desarrollar clases virtuales, realizar evaluaciones y mantener la comunicación con estudiantes, profesores y el personal administrativo. Paralelo a esta medida se brindó soporte psicológico a los estudiantes, puesto que el 85\% fue afectado por síntomas depresivos en Estados Unidos. Es por este motivo que a partir de septiembre de 2020 se inició el proceso de reapertura de las universidades, implementando sistemas mixtos de clases: presenciales y en línea.

De México tenemos el artículo Decisiones disruptivas en el entorno universitario ente la nueva realidad educativa, elaborado por José René Arroyo Ávila, María del Rosario Alvídrez Díaz y Oscar Alejandro Viramontes Olivas, que recoge las experiencias docentes en la implementación del Modelo Educativo de la Universidad Autónoma de Chihuahua y la migración de la educación presencial hacia la virtual por COVID-19. La investigación de naturaleza cualitativa da cuenta que al prohibirse reuniones sociales en México, en marzo del 2020, la Universidad Autónoma de Chihuahua inició el proceso de capacitación a sus docentes en el entorno virtual Moodle, también para ejecutar un nuevo modelo sustentado en el pensamiento complejo. Así, iniciaron la impartición de los cursos ya estandarizados en el semestre agosto-diciembre de 2020.

Magda Francisca Cejas Martínez, Mayra Fernanda Quiñonez Bedón y Juan Pablo Morales Corozco, en La formación por competencias en el docente universitario de la zona 3 del Ecuador, reportan la investigación realizada en dos universidades ecuatorianas. A través de una encuesta virtual, solicitaron a 20 docentes universitarios que identificaran las competencias que prioritariamente caracterizan al docente universitario, de una lista de 24 competencia propuestas. Concluyen que las competencias más destacables son la planificación del proceso enseñanza aprendizaje, la iniciativa, el liderazgo de equipos, pensamiento analítico y crítico, competencia comunicativa y deber social. 
En Venezuela, Eliomar Jiménez Sierra preocupado por la violencia suscitada entre los años 2016 y 2017 en la Universidad Pedagógica Experimental Libertador, Instituto Pedagógico de Barquisimeto "Luis Beltrán Prieto Figueroa", desarrolló una tesis doctoral con el método fenomenológica y en esta oportunidad nos presenta el estudio en formato artículo: Mecanismos de violencia en la universidad: Infecunda comunidad de intereses espirituales. Desde la óptica de ocho miembros de la comunidad universitaria de la UPEL-IPB, emerge la "lógica simbólica-cultural que muestra a la Universidad (deshumanizada) como un contexto de violencia, donde operan tres mecanismos de violencia: disciplinamiento, control y exclusión social” (p. 224).

Desde Colombia, Yovany Alberto Arroyave Rave expone Los saberes didácticos experienciales: reflexiones para el abordaje de una problematización, con el propósito de "reconocer la relevancia y trascendencia de los presupuestos ético-estéticos en la reconfiguración de los saberes pedagógicos y los saberes didácticos experienciales” (p. 255). El investigador precisa que siguiendo la triada dialógica hegeliana: tesis, antítesis y síntesis; a la luz de la investigación cualitativa y la hermenéutica, es posible estudiar las experiencias pedagógicas de los docentes y develar sus reconfiguraciones sobre el saber pedagógico y saber didáctico.

Por su parte, Mayra Araceli Nieves Chávez y Beatriz Elena Muñoz Serna interesadas en saber cómo se afronta emocionalmente las clases a distancia por COVID-19 en la Universidad Autónoma de Querétaro, México, desarrollaron una investigación cualitativa, bajo el nombre de La ausencia del buen humory la amorosidad en la educación a distancia. Lograron develar que existe poco de buen humor y amorosidad en las clases a distancia, porque cuesta asumir relaciones interpersonales virtuales. Sostienen que, aun cuando la experiencia sea dolorosa, se debe aliviar el desgaste del distanciamiento social, "con algo de buen humor, para acompañar amorosamente en el dolor" (p. 300).

Por su parte, Juan Carlos Araque Escalona de Ecuador presenta Breves apuntes acerca del origen del español (desde sus inicios hasta 1492): Implicaciones para una didáctica en la educación superior. En el artículo se hace un recuento histórico del español o castellano hasta la publicación de la primera gramática castellana en el año 1492 por parte de Antonio de Nebrija. Concluye que la lengua, "aunque simbólico y abstracto, configura y construye la cotidianidad de los hispanohablantes; más cuando se sabe que en ella existen términos que evolucionaron y seguirán evolucionando desde lenguas como el árabe, hebreo, vasco, alemán, inglés, francés” (p. 304) entre otras.

Isabel Teresa Suárez Pérez, María Lourdes Piñero Martín y María Giuseppina Vanga en Saberes y haceres de los docentes universitarios ante periodos de educación interrumpida, consideran que en la práctica pedagógica es necesario que los docentes universitarios desarrollen saberes y haceres para 
afrontar con éxito procesos de enseñanza en periodos de educación interrumpida por COVID-19. Guiadas por el paradigma interpretativo, método fenomenológico hermenéutico, las investigadoras recogen información mediante grupos focales y presentan los saberes y haceres enmarcados en tres áreas: “área emocional (autoconocimiento emocional o conciencia de sí mismo, automotivación, empatía o reconocimiento de las emociones ajenas, habilidades sociales), área tecnológica y área de planificación educativa" (p. 331).

Sin lugar a dudas, el libro Experiencias educativas en el contexto universitario latinoamericano se constituye en un documento a revisar para comprender la realidad universitaria en tiempos de COVID-19 y la problemática que atraviesa en la última década. 